\title{
MC-LR induced overproduction of progesterone via inhibiting miR-3473g: in vitro and in vivo evidence
}

\author{
Xiaoyan $\mathrm{Li}^{1,2, *}$, Jinling Zhu ${ }^{1,2, *}$, Jie Tian ${ }^{1,2}$, Dongmei $\mathrm{Li}^{1,2}$, Xiaodong Han ${ }^{1,2}$ and Jiang $\mathrm{Wu}^{1,2}$ \\ ${ }^{1}$ Immunology and Reproduction Biology Laboratory \& State Key Laboratory of Analytical Chemistry for Life Science, \\ Medical School, Nanjing University, Nanjing, Jiangsu, China and 'Jiangsu Key Laboratory of Molecular Medicine, \\ Medical School, Nanjing University, Nanjing, Jiangsu, China
}

Correspondence should be addressed to X Han or JWu; Email: hanxd@nju.edu.cn or wujiang@nju.edu.cn

*(X Li and $\mathrm{J}$ Zhu contributed equally to this work)

\begin{abstract}
Health risk of human exposure to microcystin-leucine arginine (MC-LR) has drawn more and more attention in recent years. In the present study, MC-LR inhibited miR-3473g expression of mouse granulosa cells both in vitro and in vivo. By dual-luciferase reporter assay, we confirmed miR-3473g is able to bind the 3'-UTR of StAR protein (StAR) mRNA and suppress StAR expression. Thus, downregulation of miR-3473g after MC-LR exposure led to StAR overexpression. Excessive StAR probably transported much more cholesterol into the inner membrane of the mitochondria and finally resulted in overproduction of progesterone. Our results revealed that MC-LR exposure was associated with premature luteinization of granulosa cells and may adversely affect women's fertility.

Reproduction (2020) 159 81-89
\end{abstract}

\section{Introduction}

Due to global warming and over-supply of nutrients (nitrogen and phosphorus) discharge from agricultural waters and wastewaters, the incidences and intensities of cyanobacterial blooms have increased substantially in the past decades (Ballot et al. 2005, Michalak et al. 2013, Paerl et al. 2014, Buratti et al. 2017). Cyanobacteria can produce a wide variety of potent toxins and pose a hazard to farming, fishing and human health (Svircev et al. 2017, Laszakovits \& MacKay 2019). Among these cyanotoxins, microcystin-leucine arginine (MC-LR) attracted most attention owing to its broadest distribution and strongest toxicity (Codd et al. 2005, Pearson et al. 2010). MC-LR is highly water stable and conventional water treatment technologies cannot effectively remove the extracellular MC-LR (Zhang et al. 2012, Jasim \& Saththasivam 2017, Jian et al. 2019). Thus, MC-LR is prone to pass into human body and impose a serious threat to humans directly through drinking water or indirectly by consumption of MC-LR contaminated aquatic organisms (Chen et al. 2018, Palagama et al. 2019).

It has been well documented that MC-LR is able to induce hepatotoxicity and neurotoxicity, kidney impairment, and gastrointestinal disorders (Wang et al. 2010, Li et al. 2015, 2019). In our previous study (Wu et al. 2015), environmental level of MC-LR was orally administered to female mice and led to decreased development of follicles, elevated serum progesterone, irregular estrous cycles and subfertility. As the basic functional unit of the ovary, each follicle is comprised of an oocyte surrounded by somatic granulosa cells. Granulosa cells are responsible for secretion of steroid hormones that are necessary to prepare the reproductive tract for fertilization and the establishment of pregnancy (Knight \& Glister 2006, Fan et al. 2009). Primary cultured mouse granulosa cells (mGCs) could uptake MC-LR and become the target of the toxicant (Wu et al. 2015).

miRNAs are endogenous noncoding RNA molecules approximately 22-25 nucleotides in length. By binding to the complementary sequences on the $3^{\prime}$-UTRs of target mRNAs resulting in mRNA translational inhibition or degradation, miRNAs play a central part in diverse cellular processes, including cell differentiation, proliferation and survival (Bartel 2004, Stark et al. 2008). Unsurprisingly, it has been reported that miRNAs play a crucial role in the female reproductive system (Carletti \& Christenson 2009, Gay et al. 2018). In order to figure out whether miRNAs participated in the toxicity of MC-LR on mGCs, we performed miRNAs microarray technology to detect the expression of miRNAs of mGCs after MC-LR exposure. The results showed that 125 miRNAs changed significantly, including 50 miRNAs downregulated and 75 miRNAs upregulated (Li et al. 2017). Among these miRNAs, the downregulated miR-3473g (fold change $>2$ ) attracted our attention. StAR protein (StAR), the crucial rate-limiting catalyzing enzymes in progesterone synthesis, was predicted to be the target gene of miR- 
3473g by miRanda (http://www.microrna.org/microrna/ home.do) and TargetScan (http://www.targetscan.org/). Downregulation of miR-3473g probably removed its inhibition on StAR and led to increased progesterone synthesis which played a key part in MC-LR-induced female reproductive toxicity. To obtain a complete understanding of abnormal progesterone synthesis after MC-LR exposure, a combined in vitro and in vivo approach was applied in the present study that focused on miR-3473g.

\section{Materials and methods \\ Ethics statement}

The animal experiments were performed according to the Guide for the Care and Use of Laboratory Animals (The Ministry of Science and Technology of China, 2006) and all experimental protocols were approved under the animal protocol number SYXK (Su) 2014-0052 issued by Jiangsu Provincial Science and Technology Department.

\section{Chemicals and reagents}

Microcystin-leucine arginine (purity $\geq 95 \%$ ) was purchased from Alexis Biochemicals (Lausen, Switzerland). One milligram MC-LR was dissolved in $100 \mu \mathrm{L}$ DMSO and diluted to $1 \mathrm{ml}$ with sterilized water as a stock solution kept at $-20^{\circ} \mathrm{C}$ within six months. Fetal bovine serum (FBS) and Dulbecco's modified Eagle's medium-Ham's F-12 (DMEM-F12) medium were purchased from Gibco. Lipofectamine 2000, SYBR GreenER qRT-PCR kits, Taqman microRNA assay and universal PCR Mastermix were from Invitrogen. Progesterone and estradiol ELISA kits were purchased from Enzo Life Sciences (Lausen, Switzerland). Rabbit anti-StAR and mouse anti-GAPDH were obtained from Abcam. Rabbit anti-luteinizing hormone/ chorionic gonadotropin receptor (anti-LHCGR) was obtained from Proteintech (Wuhan, China). Horseradish peroxidase (HRP)-conjugated goat anti-rabbit/mouse IgG, which was used as the secondary antibody, was purchased from Boster (Wuhan, China). Enhanced chemiluminescence detection kit was from Alpha (San Jose, CA, USA). Dual-Luciferase Reporter Assay System was purchased from Promega.

\section{Mouse primary granulosa cell culture and treatment}

Mouse granulosa cells were collected from the ovaries of 21-day-old immature female BALB/c mice using the follicle puncture method, as described by Kipp et al. (2007). Oocytes were filtered out with a $40-\mu \mathrm{m}$ cell strainer. The mGCs were cultured in DMEM-F12 supplemented with $10 \%$ FBS and incubated at $37^{\circ} \mathrm{C}$ with $5 \% \mathrm{CO}_{2}$ in a humidified chamber. Two days later, mGCs were adherent to the bottom of the dishes and formed a monolayer after another 2 days. The purity of mGCs obtained was above $95 \%$ when examined microscopically and the survival percentage of mGCs was above $98 \%$ assessed by in situ trypan blue staining. MC-LR (stock solution) was dissolved in DMEM-F12 and applied to mGCs at concentrations of $0,1.25,5$ and $20 \mu \mathrm{M} .48 \mathrm{~h}$ later, the RNA, protein and cell culture supernatants were harvested to detect the relative expression and hormone secretion of the cells.

For preparing miR-3473g vector transfected mGCs, transfections of miR-3473g mimic, inhibitor, control-mimic, or control-inhibitor was performed by Lipofectamine 2000. After transfection, the plate was placed in a $\mathrm{CO}_{2}$ incubator at $37^{\circ} \mathrm{C}$ for $6 \mathrm{~h}$, then the medium of mimic, inhibitor or negative control and lipo2000 mixture was removed, and fresh DMEM-F12 medium containing 10\% FBS was replaced for culture. After $24 \mathrm{~h}$ of transfection, mGCs were next incubated either in the absence or presence of $5 \mu \mathrm{M} \mathrm{MC}$-LR for $48 \mathrm{~h}$ to harvest the RNA, protein and cell culture supernatant to detect the relative expression and hormone secretion by the cells.

\section{Animals and treatment}

Six-week-old female BALB/c mice (specific pathogen-free, SPF) $(n=100)$ (18-22 g) were purchased Medical Center of Yangzhou university (Yangzhou, China). Mice were individually housed in laminar flow cabinets under a specific pathogenfree environment with access to food and water ad libitum. The animals were acclimatized for 1 week before use, and maintained throughout at standard conditions: $50 \%$ relative humidity, $24^{\circ} \mathrm{C}$, and $12: 12 \mathrm{~h}$ light-dark circle. Mice were randomized into ten groups ( $n=10$ for each) (Supplementary Table 1, see section on supplementary materials given at the end of this article). Four groups of mice were intraperitoneally injected with MC-LR at $0,7.5,22.5$ or $45 \mu \mathrm{g} / \mathrm{kg}$ body weight every day for 2 weeks. The MC-LR (stock solution) was dissolved in $400 \mu \mathrm{L}$ saline to achieve required concentrations.

Another six groups received miRNA injection according to the method of Yin et al. (2014). Mice were anesthetized using pentobarbital. A small dorsal abdominal incision was made in the lumbar region on each side of the midline. Through the incision in the dorsal abdominal wall, the ovaries and associated fat pad were gently pulled outside. The intrabursal injection was performed under microscopic magnification by inserting a 30-gauge needle with $20 \mu \mathrm{L}$ physiological saline or $2 \times 10^{6}$ transduction units of lentivirus through the ovarian fat pad into the ovarian bursa of each side. The wound was sutured with silk when the ovaries were returned to the abdominal cavity. These six groups of mice were injected with physiological saline, physiological saline, lentiviral vectors containing mimic-NC, miR-3473g-mimic, inhibitor-NC or miR-3473ginhibitor (Genechem, Shanghai, China), respectively. One week later, group 5 received intraperitoneally $400 \mu \mathrm{L}$ saline solution every day for 2 weeks and the remaining groups $6-10$ received intraperitoneally $22.5 \mu \mathrm{g} / \mathrm{kg}$ body weight MC-LR every day for 2 weeks.

\section{Quantitative RT-PCR}

Total RNA from mGCs and ovaries was isolated using TRIzol reagent (Invitrogen). The purity of RNA was determined with a spectrophotometer (Hoefer, Holliston, MA, USA). One microgram of total RNA was used for CDNA synthesis. RT was performed using the Superscript III first-strand synthesis system (Invitrogen) or the TaqMan microRNA reverse transcription kit 
(Invitrogen) on a Veriti 96-Well Fast Thermal Cycler (Applied Biosystems). Quantitative PCR was performed using the SYBR GreenER qRT-PCR kit or the Taqman microRNA assay and universal PCR Mastermix using a 7900 HT Fast Real-Time PCR System (Applied Biosystems). All the procedures were repeated for three times. For each replication of ovarian tissue, total RNA sample was extracted from one ovary of different mouse in each group. The relative quantification values for each miRNA and mRNA were calculated by the $2^{-\Delta \Delta C t}$ method using U6 and GAPDH as an internal reference, respectively. The sequences of mRNA primer pairs used in this assay included StAR, forward primer AACGGGGACGAAGTGCTAAG, reverse primer CCGTGTCTTTTCCAATCCTCTG; GAPDH, forward primer AGGTCGGTGTGAACGGATTTG, reverse primer TGTAGACCATGTAGTTGAGGTCA.

\section{Western blotting}

For in vivo part, one ovary was homogenized for extracting proteins in $1 \mathrm{~mL}$ ice-cold RIPA buffer (50 mM Tris-HCl PH 7.4, $150 \mathrm{mM} \mathrm{NaCl}, 1 \% \mathrm{NP}, 0.1 \%$ SDS, $1 \times$ phosphatase/protease inhibitors). For mGCs, $200 \mu \mathrm{L}$ protein extraction buffer was added into the dish to extract the total protein from cells. The protein concentration was determined using the BCA protein assay kit (Beyotime, Nantong, China). About $20 \mu \mathrm{g}$ of protein from each sample was separated on $10 \%$ SDS-PAGE and electrophoretically transferred to a PVDF membrane. The membrane was then blocked in TBS buffer containing $5 \%$ BSA (20 mM Tris- $\mathrm{HCl}(\mathrm{pH} \mathrm{7.6)}$ and $150 \mathrm{mM} \mathrm{NaCl})$ for $1.5 \mathrm{~h}$ at $37^{\circ} \mathrm{C}$. Next, these blots were incubated sequentially with primary rabbit anti-StAR, mouse anti-GAPDH and HRPconjugated secondary antibodies. The chromogenic signal intensity was detected using an Odyssey Scanning System (LI-COR, Lincoln, NE, USA) and quantified using ImageJ software (NIH, Bethesda, MD, USA). All the procedures were repeated for three times. For each replication of ovarian tissue, protein sample was extracted from one ovary of different mouse in each group.

\section{ELISA assay}

Progesterone and estradiol levels in cell supernatant or serum were measured by rodent ELISA kits according to the manufacturer's instructions. The detection limits were $0.1 \mathrm{ng} /$ $\mathrm{mL}$ for progesterone and $5 \mathrm{pg} / \mathrm{mL}$ for estradiol. The serum samples were prepared as described previously (Wu et al. 2015). Briefy, blood was collected by removing the eyeballs and placed in $1.5 \mathrm{~mL}$ microcentrifuge tubes at $4{ }^{\circ} \mathrm{C}$ overnight. On the second day, the tubes were centrifuged at $200 \mathrm{~g}$ for 30 $\min$ at $4^{\circ} \mathrm{C}$ and the sera were collected.

\section{Immunofluorescence}

Collected mGCs were centrifuged at $160 \mathrm{~g}$ for $5 \mathrm{~min}$ at $4{ }^{\circ} \mathrm{C}$ and incubated sequentially with $4 \%$ paraformaldehyde fixative for $10 \mathrm{~min}$ and $3 \%$ BSA-blocking agents for $30 \mathrm{~min}$. Primary monoclonal anti-StAR (1:200) was then applied and incubated overnight. Alexa Fluor ${ }^{\circledR}$ 488-conjugated goat anti-rabbit IgG $(1: 200)$ and phalloidin $(1: 1000)$ was added and samples were incubated for $1 \mathrm{~h}$ at $37^{\circ} \mathrm{C}$. Nuclei were counterstained with 5 $\mu \mathrm{g} / \mathrm{mL}$ DAPI. Finally, the slides were mounted with glycerin and observed using a confocal fluorescence microscope (Olympus).

\section{Histological evaluation and immunohistochemistry}

Ovaries were fixed in 4\% paraformaldehyde solution for 24 $\mathrm{h}$, embedded in paraffin and cut into 5- $\mu$ m-thick sections. For histological evaluation, the ovarian sections were stained with hematoxylin and eosin and observed under a light microscope DXM12000F (Nikon). For immunohistochemistry, the sections were incubated with PBS containing $3 \% \mathrm{H}_{2} \mathrm{O}_{2}$ for $10 \mathrm{~min}$ to inhibit endogenous peroxidase activity. After being blocked with $3 \%$ BSA in PBS for $1 \mathrm{~h}$ at $37^{\circ} \mathrm{C}$, the sections were subsequently incubated with rabbit anti-LHCGR (1:200) overnight at $4^{\circ} \mathrm{C}$ and then with HRP-conjugated secondary antibodies $(1: 200)$ at $37^{\circ} \mathrm{C}$ for $1 \mathrm{~h}$. HRP activity was examined using the diaminobenzidine method according to the manufacturer's instructions (Zhongshan Biotechnology, Beijing, China). After counterstaining with hematoxylin, the sections were mounted with neutral balsam for observation under a microscope DXM12000F.

\section{Dual-luciferase reporter assay}

The 3'-UTRs of mouse StAR (Supplementary Table 2) encompassing the conserved miR-3473g response elements were cloned into the GV272 vector (Shanghai Genechem Co., Ltd, China, Supplementary Fig. 1). The 293T cells were seeded in a 24-well plate for $24 \mathrm{~h}$, and transfection was performed when cell density in the plate reached 60\%. $100 \mathrm{ng}$ of each GV272-Control or GV272-StAR plasmids were used as reporter constructs and were cotransfected into 293T cells with $25 \mu \mathrm{mol}$ miRNA mimic (control mimic or miR-3473gmimic) using Lipofectamine 2000 (Invitrogen). The plate was placed in an incubator for $6 \mathrm{~h}$, and then the medium was removed and fresh complete medium was added. After $24 \mathrm{~h}$, firefly luciferase activity and Renilla activity were determined in cell lysates by Promega Dual Luciferase Assay Kit (Promega (Beijing) Biotech Co., Ltd).

\section{Statistical analysis}

SPSS 18.0 (SPSS) was used for statistical analysis. Experimental results were expressed as mean \pm S.E. The Levene's test was employed to check the normality and homogeneity of variances in the data. The Student's $t$ test was used for paired comparisons. For the comparison of three or more groups, one-way ANOVA was used for the comparison, which was followed by Duncan's post hoc test. Values of $P<0.05$ were considered statistically significant.

\section{Results}

\section{$M C-L R$-induced downregulation of miR-3473g in $m G C s$ and miR-3473g targets StAR mRNA}

We treated mGCs with $5 \mu \mathrm{M} M C$-LR for $48 \mathrm{~h}$. Then the expression of miR-3473g in mGCs was determined by 
qRT-PCR and dual-luciferase reporter assay was performed to verify the target gene of miR-3473g. The results showed that miR-3473g was significantly downregulated (Fig. 1A). Next, we tried to predict the potential targets of miR-3473g by TargetScan and miRanda. StAR stood out as one of the target mRNAs of miR-3473g. The binding sites for miR-3473g in the $3^{\prime}$-UTR of StAR were shown in Fig. 1B. Then we tried to confirm the binding of miR-3473g to the $3^{\prime}$-UTR of StAR in $293 T$ cells using the dual luciferase reporter gene assay system. StAR 3'-UTR and StAR 3'-UTR with mutations in the predicted miR-3473g binding site were cloned separately into the luciferase reporter system (GV272 vector). The GV272 vectors were then co-transfected with either miR-3473g mimic or its negative control. Luciferase activity of the system containing wildtype, but not mutated, StAR 3'-UTR was substantially decreased after co-transfection with miR-3473g mimic (Fig. 1C), which supported the prediction that StAR was the target gene of miR-3473g.

\section{MC-LR exposure led to increased StAR expression and progesterone production of $m G C s$}

Mouse granulosa cells were exposed to $0,1.25,5$ or 20 $\mu \mathrm{M}$ MC-LR for $48 \mathrm{~h}$. Quantitative RT-PCR and Western blotting were performed to detect mRNA and protein expression of StAR in mGCs. Meanwhile, progesterone secreted by mGCs in cell culture supernatants were detected by ELISA assay. The data showed that StAR

$\mathrm{B}$
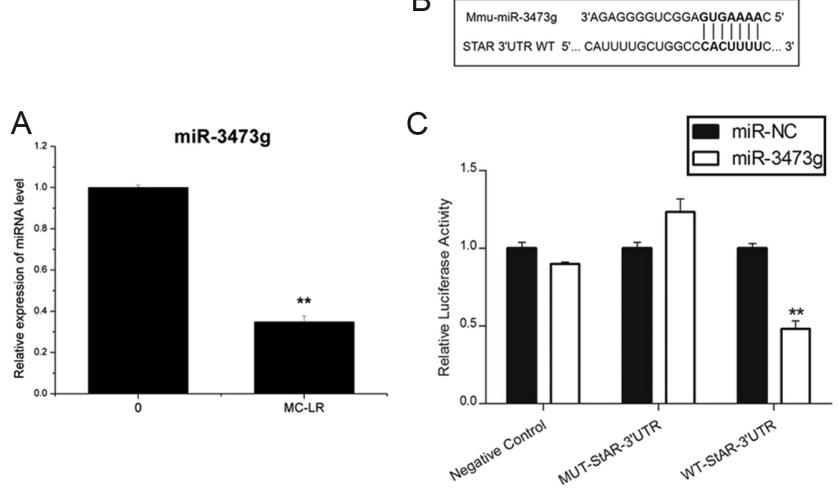

Figure 1 Effects of MC-LR on miR-3473g in mouse granulosa cells (mGCs) and validation of miR-3473g target gene by luciferase reporter assay. (A) miR-3473g expression in mGCs after 48-h MC-LR exposure at $5 \mu \mathrm{M}$ was measured by qRT-PCR. (B) Nucleotide sequences of murine miR-3473g and the 3'-UTR of StAR region, which encompasses the miR-3473g seed match region (bold). (C) Luciferase reporter assays were carried out in 293T cells following co-transfection of miR-3473g mimic negative control (miR-NC) or miR-3473g mimic (miR-3473g) together with empty vector (negative control), mutant (MUT) StAR vector or wild-type (WT) StAR vector, as indicated. Firefly luciferase activity was normalized based on the Renilla luciferase activity. Data are shown as mean \pm S.E. $(n=3)$. $* * P<0.01$
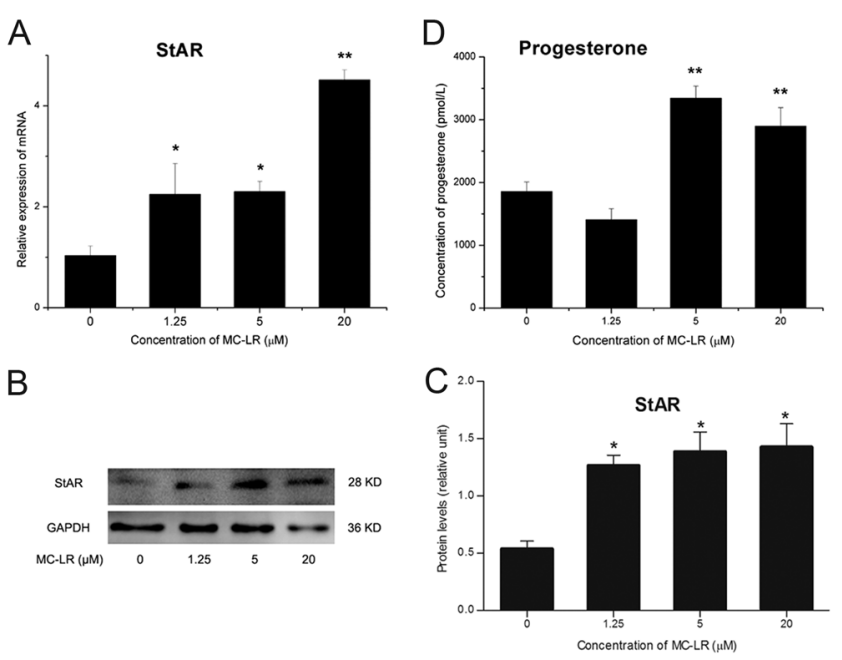

Figure 2 Effects of MC-LR on StAR expression and progesterone production of mouse granulosa cells (mGCs). Granulosa cells were exposed to $0,1.25,5$ or $20 \mu \mathrm{M}$ MC-LR for $48 \mathrm{~h}$. (A) The mRNA levels of StAR in mGCs were determined by qRT-PCR. (B and C) The protein levels of StAR in mGCs were determined by Western blotting (B) and densitometry data are shown (C). (D) The concentrations of progesterone in $\mathrm{mGCs}$ cell culture supernatants were determined by ELISA assay after 48-h MC-LR exposure. Data are shown as mean \pm S.E. $(n=3) .{ }^{*} P<0.05, * * P<0.01$.

mRNA and protein in all the MC-LR-treated groups were significantly enhanced (Fig. 2A, B and C). As StAR is the key rate-limiting enzyme in progesterone synthesis, we can reasonably expect the change of progesterone concentration in mGCs cell culture supernatants. The average progesterone concentration in control group was $1855 \pm 151 \mathrm{pmol} / \mathrm{L}$, while values in experimental groups treated with $1.25,5$ and $20 \mu \mathrm{M} \mathrm{MC-LR}$ were $1410 \pm 175,3344 \pm 192$ and $2896 \pm 298 \mathrm{pmol} / \mathrm{L}$. The coefficients of variation were 14.1, 21.5, 9.9 and $17.8 \%$, respectively. Compared with the control group, progesterone production significantly increased in 5 and $20 \mu \mathrm{M}$ MC-LR-treated groups (Fig. 2D).

\section{Over-expression of miR-3473g relieved $M C$-LR-induced upregulation of StAR expression and progesterone production in vitro}

To examine the regulatory effects of miR-3473g on progesterone synthesis of mGCs, we transfected mGCs with miR-3473g-mimic, miR-3473g-inhibitor, and their negative controls followed by exposure to $5 \mu \mathrm{M}$ MC-LR for $48 \mathrm{~h}$. The transfection had no significantly effect on cell viability of mGCs (Supplementary Fig. 2). Quantitative RT-PCR was performed to detect miR3473g and STAR mRNA in mGCs. Western blotting and immunofluorescence were used to detect STAR protein in mGCs. Progesterone secreted by mGCs were detected in cell culture supernatants by ELISA assay. Figure 3A showed that miR-3473g was highly increased in mGCs transfected with miR-3473g mimic and decreased 
in mGCs transfected with miR-3473g inhibitor. The expression of StAR mRNA of MC-LR-treated mGCs was suppressed by miR-3473g-mimic and remained high in miR-3473g-inhibitor group (Fig. 3B). The expression of StAR protein showed the same trend as mRNA (Fig. 3C, $\mathrm{D}$ and $\mathrm{E})$. At the same time, miR-3473g mimic could reverse MC-LR-induced progesterone production of mGCs (Fig. 3F). Elevated progesterone often comes from premature luteinization of granulosa cells accompanied by a increase in ratio of progesterone to estradiol (P:E ratio). Not surprisingly, P:E ratio in MC-LR group was much higher compared with control group which indicated that mGCs exhibited signs of premature

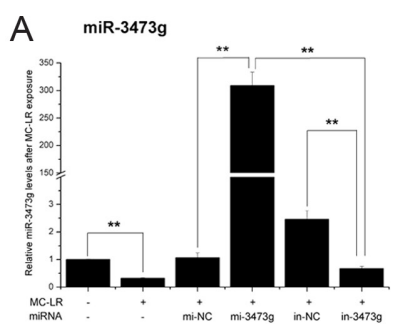

B

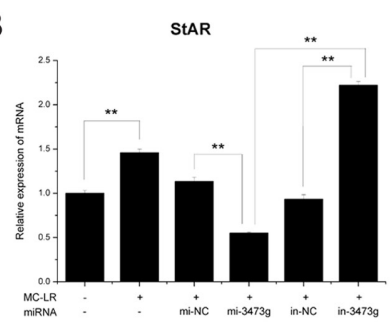

C

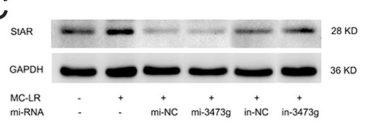

E

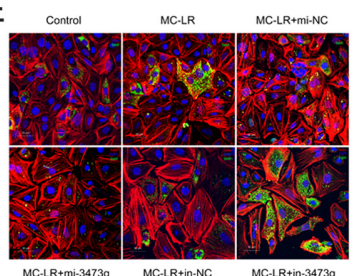

G

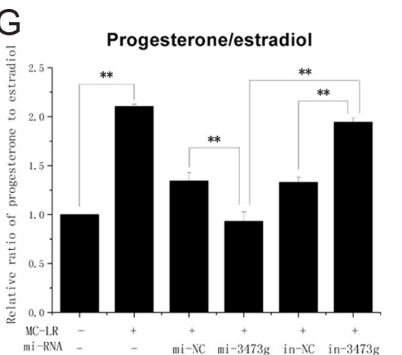

D

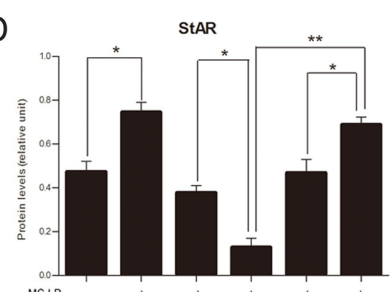

MC-LR
MiRNA

F

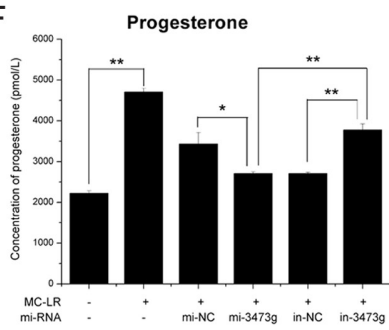

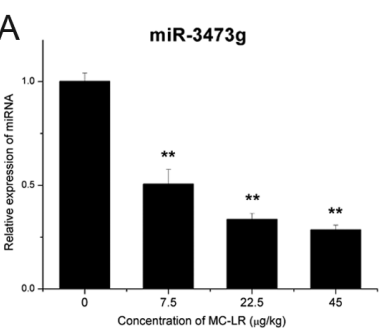

C

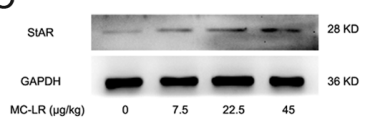

E

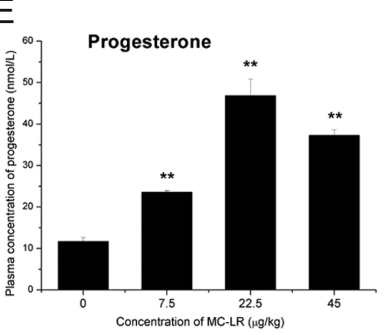

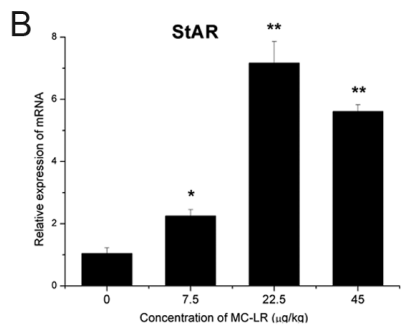

D StAR

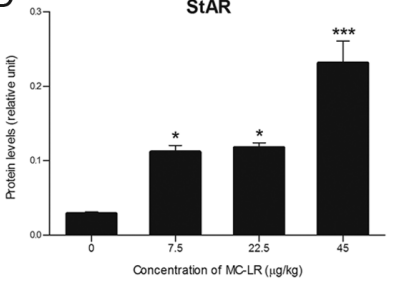

Figure 4 The effects of MC-LR on expression of miR-3473g, StAR in mouse ovaries and serum progesterone. Mice were intraperitoneally injected with MC-LR at 0, 7.5, 22.5 or $45 \mu \mathrm{g} / \mathrm{kg}$ body weight every day for 2 weeks. (A) The expression of miR-3473g in ovarian tissues was measured by qRT-PCR. (B) The StAR mRNA in ovarian tissues were measured by qRT-PCR. (C and D) The StAR protein in ovarian tissues were measured by Western blotting $(C)$ and densitometry data are shown (D). (E) The concentrations of serum progesterone were determined by ELISA assay. Data are shown as mean \pm S.E. $(n=3)$. $* P<0.05, * * P<0.01$.

luteinization. Transfection of miR-3473g mimic prevented the increase of P:E ratio (Fig. 3G).

\section{MC-LR inhibited miR-3473g and led to increased StAR and progesterone in vivo}

Four groups of mice ( $n=10$ for each) were intraperitoneally injected with MC-LR at $0,7.5,22.5$ or $45 \mu \mathrm{g} / \mathrm{kg}$ body weight every day. Two weeks later, the expression of miR-3473g was determined in ovarian tissues. miR-3473g was significantly downregulated in a dose-dependent manner (Fig. 4A). We next analyzed StAR expression at the mRNA and protein levels (Fig. $4 B, C$ and D) and the results showed a significant upregulation. However, maximum increment was not observed at the high dose MC-LR treatment group. The high dose of MC-LR may cause other damages besides inhibition of miR-3473g. The average serum progesterone concentrations of $0,7.5,22.5$ or $45 \mu \mathrm{g} / \mathrm{kg}$ MC-LR-treated mice were $11.69 \pm 0.98,23.58 \pm 0.33$, $46.85 \pm 3.95$ and $37.28 \pm 1.32 \mathrm{pmol} / \mathrm{L}$. The coefficients of variation were $14.5,2.5,14.6$ and $6.1 \%$, respectively (Fig. 4E). These results were consistent with our in vitro findings. 
Over-expression of miR-3473g held back MC-LRinduced upregulation of StAR and progesterone in vivo

Six groups of mice ( $n=10$ for each) received intraperitoneal injection of physiological saline, physiological saline, lentiviral vectors containing mimic-NC, miR-3473g-mimic, inhibitor-NC or miR$3473 \mathrm{~g}$-inhibitor, respectively. One week later, the control group received intraperitoneally $400 \mu \mathrm{L}$ saline solution every day for 2 weeks and the remaining five groups received intraperitoneally $22.5 \mu \mathrm{g} / \mathrm{kg}$ body weight MC-LR every day for 2 weeks. We found that the mimic increased the expression of miR-3473g, whereas the inhibitor suppressed the expression of miR-3473g (Fig. $5 \mathrm{~A})$. We further investigated the effect of manipulated miR-3473g expression on the ovarian StAR and serum progesterone with or without MC-LR exposure. Overexpression of miR-3473g could inhibit the upregulation of StAR induced by MC-LR at both mRNA and protein levels (Fig. 5B, C and D). As the consequence, serum progesterone also declined (Fig. 5E).

\section{MC-LR induced upregulation of LHCGR and premature luteinization of mGCs in ovary which could be reversed by $\mathrm{miR}-3473 \mathrm{~g}$}

The remaining ovaries of six groups of mice in Fig. 5 were taken for LHCGR detection and histological evaluation. Compared with control, LHCGR expression of the follicular mGCs was significantly upregulated in MC-LR-treated group. Meanwhile, the upregulation of LHCGR induced by MC-LR was inhibited by miR-3473g (Fig. 6A). As Fig. 6B showed, in the control or combined treatment of MC-LR and mi-3473g groups, the mGCs of antral follicles exhibited normal morphology. By contrast, in the remaining four groups, mGCs of antral follicles presented a clear sign of luteinization. Specifically, the connection between granulosa cells got loose and small round vacuoles appeared. The staining of mGCs nucleus faded and round lipid droplets formed in the cytoplasm of mGCs. These changes demonstrated that MC-LR exposure did trigger the premature luteinization of mGCs and mi-3473g played a pivotal role in this process.

\section{Discussion}

MC-LR has triggered increasing concerns about the health risk due to its global distribution. As relatively large hydrophilic molecules, MC-LR enters cells with the assistance of specific membrane transporters named organic anion transporter peptides (OATPs) (Fischer et al. 2005). Following cell entry, MC-LR can cause toxic effects through multiple cellular pathways that may differ according to the duration and approach of exposure. It has been well documented that MC-LR inhibits serine/ threonine protein phosphatases (PP) and results in a set
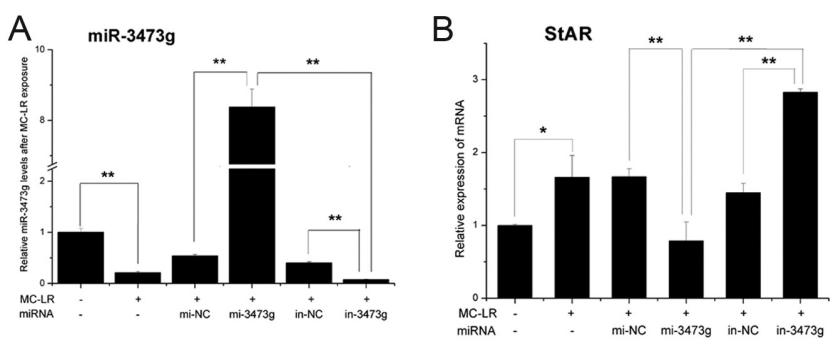

C
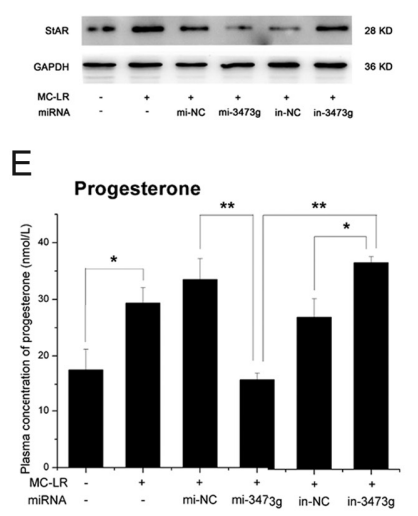

D

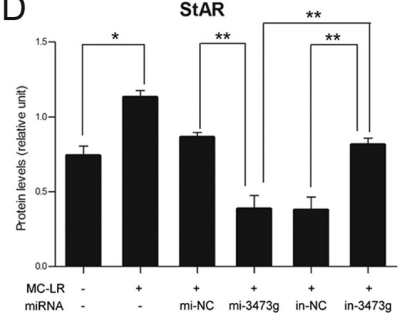

Figure 5 Validating the functions of miR-3473g on ovarian StAR and serum progesterone levels of female mouse. Mice received intraperitoneal injection of physiological saline, physiological saline, lentiviral vectors containing mimic-NC, miR-3473g-mimic, inhibitor-NC or miR-3473g-inhibitor, respectively. One week later, the control group received intraperitoneally $400 \mu \mathrm{L}$ saline solution every day for 2 weeks and the remaining five groups received intraperitoneally $22.5 \mu \mathrm{g} / \mathrm{kg}$ body weight MC-LR every day for 2 weeks. (A) The expression of miR-3473g in ovarian tissues was measured by qRT-PCR. (B) The StAR mRNA in ovarian tissues were measured by qRT-PCR. (C and D) The StAR protein in ovarian tissues were measured by Western blotting $(C)$ and densitometry data are shown (D). (E) The concentrations of serum progesterone were determined by ELISA assay. Data are shown as mean \pm S.E. $(n=3)$. $* P<0.05, * * P<0.01$.

of negative effects (Zhou et al. 2015). Oxidative stress was often observed due to overproduction of reactive oxygen species or depletion of glutathione in many MC-LR exposure studies (Shi 2009, Puerto et al. 2010, Meng et al. 2015). DNA damage occurred after MC-LR exposure resulting from either oxidative damage (Zegura et al. 2011) or direct interference to DNA damage repair pathways (Zegura et al. 2008). MC-LR has been found to distribute in the liver, kidneys, lungs, brain, heart, gonads and is able to accumulate in organs by low-dose exposures during the whole life (Wang et al. 2008). Dozens of studies focused on male reproductive toxicity of MC-LR. Decrease in testis weight, sperm concentration, level of serum testosterone and damage to spermatogenic epithelium appeared after MC-LR exposure (Li et al. 2008, Wang et al. 2013). There are also some reports about reproductive toxicity of MC-LR on female fish (Kawan et al. 2019, Liu et al. 2019), but the reports of its female mammalian reproductive toxicity 
A
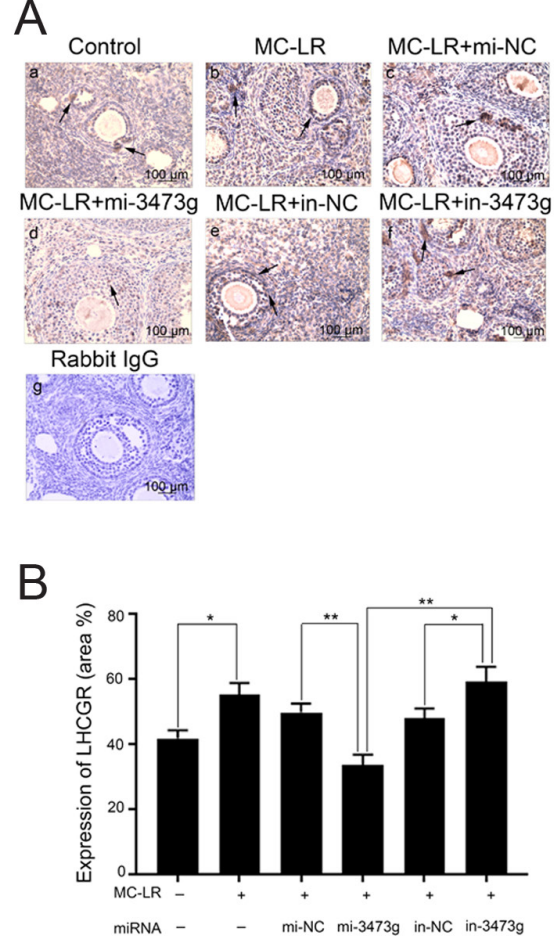
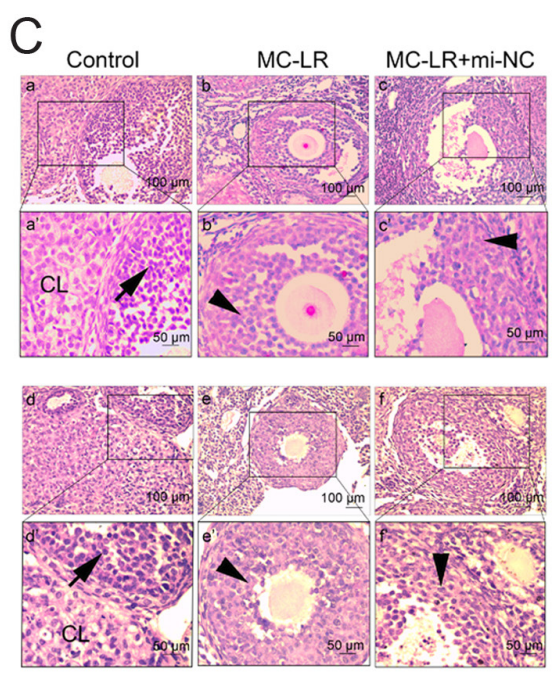

MC-LR+mi-3473g MC-LR+in-NC MC-LR+in-3473g

Figure 6 Validating the functions of miR-3473g on luteinizing hormone/chorionic gonadotropin receptor (LHCGR) level and premature luteinization of granulosa cells in mouse ovary. Mice received intraperitoneal injection of physiological saline, physiological saline, lentiviral vectors containing mimic-NC, miR-3473g-mimic, inhibitor-NC or miR3473g-inhibitor, respectively. One week later, the control group received intraperitoneally $400 \mu \mathrm{L}$ saline solution every day for 2 weeks and the remaining five groups received intraperitoneally $22.5 \mu \mathrm{g} / \mathrm{kg}$ body weight MC-LR every day for 2 weeks. (A) Representative images of LHCGR expression patterns in mouse ovarian tissues determined by immunohistochemistry. The LHCGRpositive cells are in brown (arrow) and the negative cells are in blue. (B) Quantitative analysis of LHCGR expression in ovary using ImageJ software. Data are shown as mean \pm S.E. $(n=3) . * P<0.05,{ }^{* *} P<0.01$. (C) The ovarian histological analysis by hematoxylin-eosin staining shows abnormal premature luteinization of granulosa cells, normal granulosa cells (arrow), luteinized granulosa cells (arrow head), corpus luteum (CL).

are relatively few. Our previous research demonstrated female reproductive toxicity of MC-LR on mice and first discovered that 125 miRNAs of mGCs significantly changed after MC-LR exposure (Wu et al. 2015, Li et al. 2017). In the present study, we confirmed that miR$3473 \mathrm{~g}$ in mGCs was downregulated after treatment of MC-LR. By dual-luciferase reporter assay, we established that miR-3473g was able to bind 3'-UTRs of mouse StAR and inhibited StAR expression.

The first step of steroidogenesis occurs within mitochondria. The principal action of StAR is to facilitate the movement of cholesterol from the outer mitochondrial membrane to the inner mitochondrial membrane (Stocco \& Clark 1996, Christenson \& Strauss 2000). In the inner mitochondrial membrane, cholesterol becomes substrate of P450scc and is converted to pregnenolone (Simpson \& Boyd 1966, Miller 2017). Afterward, pregnenolone is catalyzed by a $42-\mathrm{kDa}$

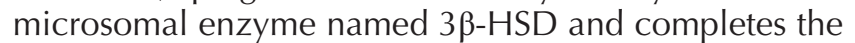
conversion to progesterone (Miller \& Auchus 2011).

Following ovulation, luteinized granulosa cells secrete progesterone to establish and maintain early pregnancy (Sellix 2015, Skory et al. 2015). However, in the previous follicular phase, a selected cohort of antral follicles grow rapidly. Their steroidogenetic activity and response to gonadotrophins are finely modulated to prevent premature luteinization. The inhibition of premature luteinization is required to sensitize certain follicles for further growth and selection of a dominant follicle (Oktem \& Urman 2010). Premature luteinization characterized by elevated progesterone in the late follicular phase has been well reported in the past
30 years. It was supposed to affect $12.3-46.7 \%$ of in vitro fertilization cycles depending on progesterone (Venetis et al. 2013). Endometrial receptivity relies heavily on the duration of progesterone exposure after adequate estrogen exposition. Premature progesterone elevation in patients was demonstrated to affect gene expression profiles in the endometrium and disrupt endometrial receptivity (Labarta et al. 2011, Van Vaerenbergh et al. 2011). Due to high progesterone, altered epigenetic modification status found in the endometrium may pose a negative impact on endometrial receptivity and lead to lower pregnancy rates (Xiong et al. 2017). Furthermore, recent studies reported that elevated progesterone toward the end of the follicular phase had a negative influence on the oocyte or embryo quality (Huang et al. 2016, Vanni et al. 2017).

To date, the exact cause of progesterone elevation in the late follicular phase is not completely clear (Lawrenz et al. 2018). There was a persistent and rapid decline in reproductive functions in recent years. Environmental chemicals have been considered as a potential risk factor contributing to increasing reproductive dysfunction (Messerlian et al. 2018, Hipwell et al. 2019, Kim et al. 2019). Our current study demonstrated that MC-LR exposure induced the elevation of progesterone accompanied by an increase in P:E ratio. We also observed evident upregulation of LHCGR expression and premature luteinization of mGCs in ovaries of MC-LRtreated mice. Downregulation of miR-3473g was a key point for the MC-LR-induced progesterone elevation and premature luteinization of mGCs. Our findings revealed that MC-LR was able to cause progesterone elevation 
associated with premature luteinization and threaten female reproductive capacity.

In conclusion, our results indicated that MC-LR could inhibit miR-3473g of granulosa cells both in vitro and in vivo. As the target of miR-3473g, StAR was significantly upregulated after MC-LR exposure. Excessive StAR probably transported much more cholesterol into the inner membrane of the mitochondria and finally led to overproduction of progesterone. Therefore, we demonstrated that MC-LR exposure is correlated with premature luteinization of granulosa cells and poses a serious threat on reproductive health of women.

\section{Supplementary materials}

This is linked to the online version of the paper at https://doi.org/10.1530/REP-19-0313.

\section{Declaration of interest}

The authors declare that there is no conflict of interest that could be perceived as prejudicing the impartiality of the research reported.

\section{Funding}

This work was supported by National Natural Science Foundation of China (31670519, 31870492, 31901182 and 31971517) and Fundamental Research Funds for the Central Universities (0214-14380438).

\section{Author contribution statement}

Conceptualization: J W and X H; Funding acquisition: J W and $X \mathrm{H}$; Project administration: $X \mathrm{~L}, \mathrm{~J} Z$ and $\mathrm{J} T$; Writing - original draft: $X$ L, J Z and J W; Writing - review and editing: J W, $\mathrm{D} L$ and $X \mathrm{H}$.

\section{References}

Ballot A, Krienitz L, Kotut K, Wiegand C \& Pflugmacher S 2005 Cyanobacteria and cyanobacterial toxins in the alkaline crater Lakes Sonachi and Simbi, Kenya. Harmful Algae 4 139-150. (https://doi. org/10.1016/j.hal.2004.01.001)

Bartel DP 2004 MicroRNAs: genomics, biogenesis, mechanism, and function. Cell 116 281-297. (https://doi.org/10.1016/s0092-8674(04)00045-5)

Buratti FM, Manganelli M, Vichi S, Stefanelli M, Scardala S, Testai E \& Funari E 2017 Cyanotoxins: producing organisms, occurrence, toxicity, mechanism of action and human health toxicological risk evaluation. Archives of Toxicology 91 1049-1130. (https://doi.org/10.1007/s00204016-1913-6)

Carletti MZ \& Christenson LK 2009 MicroRNA in the ovary and female reproductive tract. Journal of Animal Science 87 E29-E38. (https://doi. org/10.2527/jas.2008-1331)

Chen L, Wang Y, Giesy JP, Chen F, Shi T, Chen J \& Xie P 2018 MicrocystinLR affects the hypothalamic-pituitary-inter-renal (HPI) axis in early life stages (embryos and larvae) of zebrafish. Environmental Pollution 241 540-548. (https://doi.org/10.1016/j.envpol.2018.05.024)

Christenson LK \& Strauss JF 2000 Steroidogenic acute regulatory protein (StAR) and the intramitochondrial translocation of cholesterol.
Biochimica et Biophysica Acta 1529 175-187. (https://doi.org/10.1016/ s1388-1981(00)00147-5)

Codd GA, Morrison LF \& Metcalf JS 2005 Cyanobacterial toxins: risk management for health protection. Toxicology and Applied Pharmacology 203 264-272. (https://doi.org/10.1016/j.taap.2004.02.016)

Fan HY, Liu ZL, Shimada M, Sterneck E, Johnson PF, Hedrick SM \& Richards JS 2009 MAPK3/1 (ERK1/2) in ovarian granulosa cells are essential for female fertility. Science 324 938-941. (https://doi. org/10.1126/science.1171396)

Fischer WJ, Altheimer S, Cattori V, Meier PJ, Dietrich DR \& Hagenbuch B 2005 Organic anion transporting polypeptides expressed in liver and brain mediate uptake of microcystin. Toxicology and Applied Pharmacology 203 257-263. (https://doi.org/10.1016/j.taap.2004.08.012)

Gay S, Bugeon J, Bouchareb A, Henry L, Delahaye C, Legeai F, Montfort J, Le Cam A, Siegel A, Bobe J et al. 2018 MiR-202 controls female fecundity by regulating medaka oogenesis. PLoS Genetics 14 e1007593. (https://doi.org/10.1371/journal.pgen.1007593)

Hipwell AE, Kahn LG, Factor-Litvak P, Porucznik CA, Siegel EL, Fichorova RN, Hamman RF, Klein-Fedyshin M, Harley KG \& program collaborators for Environmental influences on Child Health Outcomes 2019 Exposure to non-persistent chemicals in consumer products and fecundability: a systematic review. Human Reproduction Update $\mathbf{2 5}$ 51-71. (https://doi.org/10.1093/humupd/dmy032)

Huang B, Ren X, Wu L, Zhu L, Xu B, Li Y, Ai J \& Jin L 2016 Elevated progesterone levels on the day of oocyte maturation may affect top quality embryo IVF cycles. PLOS ONE 11 e0145895. (https://doi. org/10.1371/journal.pone.0145895)

Jasim SY \& Saththasivam J 2017 Advanced oxidation processes to remove cyanotoxins in water. Desalination 406 83-87. (https://doi.org/10.1016/j. desal.2016.06.031)

Jian ZY, Bai YH, Chang YY, Liang JS \& Qu JH 2019 Removal of micropollutants and cyanobacteria from drinking water using $\mathrm{KMnO} 4$ pre-oxidation coupled with bioaugmentation. Chemosphere 215 1-7. (https://doi.org/10.1016/j.chemosphere.2018.10.013)

Kawan A, Zhang T, Liu W, Mukhtar H, Zhan C \& Zhang X 2019 Recovery of reproductive function of female zebrafish from the toxic effects of microcystin-LR exposure. Aquatic Toxicology 214 105240. (https://doi. org/10.1016/j.aquatox.2019.105240)

Kim YR, Pacella RE, Harden FA, White N \& Toms LL 2019 A systematic review: impact of endocrine disrupting chemicals exposure on fecundity as measured by time to pregnancy. Environmental Research 171 119-133. (https://doi.org/10.1016/j.envres.2018.12.065)

Kipp JL, Kilen SM, Woodruff TK \& Mayo KE 2007 Activin regulates estrogen receptor gene expression in the mouse ovary. Journal of Biological Chemistry 282 36755-36765. (https://doi.org/10.1074/jbc.M705143200)

Knight PG \& Glister C 2006 TGF-beta superfamily members and ovarian follicle development. Reproduction 132 191-206. (https://doi. org/10.1530/rep.1.01074)

Labarta E, Martinez-Conejero JA, Alama P, Horcajadas JA, Pellicer A, Simon C \& Bosch E 2011 Endometrial receptivity is affected in women with high circulating progesterone levels at the end of the follicular phase: a functional genomics analysis. Human Reproduction 26 1813-1825. (https://doi.org/10.1093/humrep/der126)

Laszakovits JR \& MacKay AA 2019 Removal of cyanotoxins by potassium permanganate: incorporating competition from natural water constituents. Water Research 155 86-95. (https://doi.org/10.1016/j. watres.2019.02.018)

Lawrenz B, Melado L \& Fatemi H 2018 Premature progesterone rise in ART-cycles. Reproductive Biology 18 1-4. (https://doi.org/10.1016/j. repbio.2018.01.001)

Li Y, Sheng J, Sha J \& Han X 2008 The toxic effects of microcystinLR on the reproductive system of male rats in vivo and in vitro. Reproductive Toxicology 26 239-245. (https://doi.org/10.1016/j. reprotox.2008.09.004)

Li X, Zhao Q, Zhou W, Xu L \& Wang Y 2015 Effects of chronic exposure to microcystin-LR on hepatocyte mitochondrial DNA replication in mice. Environmental Science and Technology 49 4665-4672. (https://doi. org/10.1021/es5059132)

Li XY, Zhuang X, Xu TC, Mao M, Wang C, Chen YB, Han XD \& Wu J 2017 Expression analysis of microRNAs and mRNAs in ovarian granulosa cells after microcystin-LR exposure. Toxicon 129 11-19. (https://doi. org/10.1016/j.toxicon.2017.01.022) 
Li J, Chen CY, Zhang TZ, Liu WJ, Wang L, Chen YY, Wu L, Hegazy AM, El-Sayed AF \& Zhang XZ 2019 mu Evaluation of microcystin-LR absorption using an in vivo intestine model and its effect on zebrafish intestine. Aquatic Toxicology 206 186-194. (https://doi.org/10.1016/j. aquatox.2018.11.014)

Liu W, Zhan C, Zhang T \& Zhang X 2019 Microcystin-LR influences the in vitro oocyte maturation of zebrafish by activating the MAPK pathway. Aquatic Toxicology 215 105261. (https://doi.org/10.1016/j. aquatox.2019.105261)

Meng GM, Liu JH, Lin SY, Guo ZL \& Xu LH 2015 Microcystin-LR-caused ROS generation involved in $\mathrm{p} 38$ activation and tau hyperphosphorylation in neuroendocrine (PC12) cells. Environmental Toxicology 30 366-374. (https://doi.org/10.1002/tox.21914)

Messerlian C, Williams PL, Minguez-Alarcon L, Carignan CC, Ford JB, Butt CM, Meeker JD, Stapleton HM, Souter I, Hauser R et al. 2018 Organophosphate flame-retardant metabolite concentrations and pregnancy loss among women conceiving with assisted reproductive technology. Fertility and Sterility 110 1137.e1-1144.e1. (https://doi. org/10.1016/j.fertnstert.2018.06.045)

Michalak AM, Anderson EJ, Beletsky D, Boland S, Bosch NS, Bridgeman TB, Chaffin JD, Cho K, Confesor R, Daloglu I et al. 2013 Record-setting algal bloom in Lake Erie caused by agricultural and meteorological trends consistent with expected future conditions. PNAS $\mathbf{1 1 0}$ 6448-6452. (https://doi.org/10.1073/pnas.1216006110)

Miller WL 2017 Steroidogenesis: unanswered questions. Trends in Endocrinology and Metabolism 28 771-793. (https://doi.org/10.1016/j. tem.2017.09.002)

Miller WL \& Auchus RJ 2011 The molecular biology, biochemistry, and physiology of human steroidogenesis and its disorders. Endocrine Reviews 32 81-151. (https://doi.org/10.1210/er.2010-0013)

Oktem O \& Urman B 2010 Understanding follicle growth in vivo. Human Reproduction 25 2944-2954. (https://doi.org/10.1093/humrep/deq275)

Paerl HW, Gardner WS, McCarthy MJ, Peierls BL \& Wilhelm SW 2014 Algal blooms: noteworthy nitrogen. Science 346 175-175. (https://doi. org/10.1126/science.346.6206.175-a)

Palagama DSW, Devasurendra AM, Baliu-Rodriguez D, Kirchhoff JR \& Isailovic D 2019 Treated rice husk as a recyclable sorbent for the removal of microcystins from water. Science of the Total Environment 666 1292-1300. (https://doi.org/10.1016/j.scitotenv.2019.02.042)

Pearson L, Mihali T, Moffitt M, Kellmann R \& Neilan B 2010 On the chemistry, toxicology and genetics of the cyanobacterial toxins, microcystin, nodularin, saxitoxin and cylindrospermopsin. Marine Drugs 8 1650-1680. (https://doi.org/10.3390/md8051650)

Puerto M, Pichardo S, Jos A, Prieto AI, Sevilla E, Frias JE \& Camean AM 2010 Differential oxidative stress responses to pure microcystin-LR and microcystin-containing and non-containing cyanobacterial crude extracts on Caco-2 cells. Toxicon 55 514-522. (https://doi.org/10.1016/j. toxicon.2009.10.003)

Sellix MT 2015 Circadian clock function in the mammalian ovary. Journal of Biological Rhythms 30 7-19. (https://doi. org/10.1177/0748730414554222)

Shi YG 2009 Serine/threonine phosphatases: mechanism through structure. Cell 139 468-484. (https://doi.org/10.1016/j.cell.2009.10.006)

Simpson ER \& Boyd GS 1966 The cholesterol side-chain cleavage system of the adrenal cortex: a mixed-function oxidase. Biochemical and Biophysical Research Communications 24 10-17. (https://doi. org/10.1016/0006-291x(66)90402-5)

Skory RM, Xu YM, Shea LD \& Woodruff TK 2015 Engineering the ovarian cycle using in vitro follicle culture. Human Reproduction $\mathbf{3 0}$ 1386-1395. (https://doi.org/10.1093/humrep/dev052)

Stark A, Bushati N, Jan CH, Kheradpour P, Hodges E, Brennecke J, Bartel DP, Cohen SM \& Kellis M 2008 A single Hox locus in Drosophila produces functional microRNAs from opposite DNA strands. Genes and Development 22 8-13. (https://doi.org/10.1101/gad.1613108)

Stocco DM \& Clark BJ 1996 Regulation of the acute production of steroids in steroidogenic cells. Endocrine Reviews 17 221-244. (https://doi. org/10.1210/edrv-17-3-221)
Svircev Z, Drobac D, Tokodi N, Mijovic B, Codd GA \& Meriluoto J 2017 Toxicology of microcystins with reference to cases of human intoxications and epidemiological investigations of exposures to cyanobacteria and cyanotoxins. Archives of Toxicology 91 621-650. (https://doi.org/10.1007/s00204-016-1921-6)

Van Vaerenbergh I, Fatemi HM, Blockeel C, Van Lommel L, In't Veld P, Schuit F, Kolibianakis EM, Devroey P \& Bourgain C 2011 Progesterone rise on HCG day in $\mathrm{GnRH}$ antagonist/rFSH stimulated cycles affects endometrial gene expression. Reproductive Biomedicine Online 22 263-271. (https://doi.org/10.1016/j.rbmo.2010.11.002)

Vanni VS, Somigliana E, Reschini M, Pagliardini L, Marotta E, Faulisi S, Paffoni A, Vigano P, Vegetti W, Candiani M et al. 2017 Top quality blastocyst formation rates in relation to progesterone levels on the day of oocyte maturation in GnRH antagonist IVF/ICSI cycles. PLOS ONE 12 e0176482. (https://doi.org/10.1371/journal.pone.0176482)

Venetis CA, Kolibianakis EM, Bosdou JK \& Tarlatzis BC 2013 Progesterone elevation and probability of pregnancy after IVF: a systematic review and meta-analysis of over 60000 cycles. Human Reproduction Update 19 433-457. (https://doi.org/10.1093/humupd/dmt014)

Wang Q, Xie P, Chen J \& Liang G 2008 Distribution of microcystins in various organs (heart, liver, intestine, gonad, brain, kidney and lung) of Wistar rat via intravenous injection. Toxicon 52 721-727. (https://doi. org/10.1016/j.toxicon.2008.08.004)

Wang M, Wang D, Lin L \& Hong H 2010 Protein profiles in zebrafish (Danio rerio) brains exposed to chronic microcystin-LR. Chemosphere 81 716-724. (https://doi.org/10.1016/j.chemosphere.2010.07.061)

Wang X, Chen Y, Zuo X, Ding N, Zeng H, Zou X \& Han X 2013 Microcystin (-LR) induced testicular cell apoptosis via up-regulating apoptosis-related genes in vivo. Food and Chemical Toxicology 60 309-317. (https://doi. org/10.1016/j.fct.2013.07.039)

Wu J, Yuan MM, Song YF, Sun F \& Han XD 2015 MC-LR exposure leads to subfertility of female mice and induces oxidative stress in granulosa cells. Toxins 7 5212-5223. (https://doi.org/10.3390/toxins7124872)

Xiong Y, Wang J, Liu L, Chen X, Xu H, Li TC, Wang CC \& Zhang S 2017 Effects of high progesterone level on the day of human chorionic gonadotrophin administration in in vitro fertilization cycles on epigenetic modification of endometrium in the peri-implantation period. Fertility and Sterility 108 269.e1-276.e1. (https://doi.org/10.1016/j.fertnstert.2017.06.004)

Yin MM, Wang XR, Yao GD, Lu MR, Liang M, Sun YP \& Sun F 2014 Transactivation of microRNA-320 by microRNA-383 regulates granulosa cell functions by targeting E2F1 and SF-1 proteins. Journal of Biological Chemistry 289 18239-18257. (https://doi.org/10.1074/jbc. M113.546044)

Zegura B, Zajc I, Lah TT \& Filipic M 2008 Patterns of microcystin-LR induced alteration of the expression of genes involved in response to DNA damage and apoptosis. Toxicon 51 615-623. (https://doi. org/10.1016/j.toxicon.2007.11.009)

Zegura B, Straser A \& Filipic M 2011 Genotoxicity and potential carcinogenicity of cyanobacterial toxins - a review. Mutation Research 727 16-41. (https://doi.org/10.1016/j.mrrev.2011.01.002)

Zhang H, Huang Q, Ke ZG, Yang LF, Wang XQ \& Yu Z 2012 Degradation of microcystin-LR in water by glow discharge plasma oxidation at the gas-solution interface and its safety evaluation. Water Research $\mathbf{4 6}$ 6554-6562. (https://doi.org/10.1016/j.watres.2012.09.041)

Zhou M, Tu WW \& Xu J 2015 Mechanisms of microcystin-LR-induced cytoskeletal disruption in animal cells. Toxicon 101 92-100. (https://doi. org/10.1016/j.toxicon.2015.05.005)

Received 15 July 2019

First decision 31 July 2019

Revised manuscript received 6 November 2019

Accepted 11 November 2019 